\title{
Pathophysiology of Osgood-Schlatter Disease: Does Vitamin D have a Role?
}

\author{
Smida $\mathbf{M}^{1^{*}}$, Kandara $\mathbf{H}^{2}$, Jlalia $\mathbf{Z}^{1}$ and Saied $\mathbf{W}^{3}$
}

${ }^{1}$ Orthopedics Kassab Institute, La Manouba, Tunis Faculty of Medicine, Manar University, Tunisia

${ }^{2}$ National Nutrition Institute, Bab Saadoun, Tunis, Tunis Faculty of Medicine, Manar University, Tunisia

${ }^{3}$ Tunis Children Hospital, Bab Saadoun, Tunis, Tunis Faculty of Medicine, Manar University, Tunisia

"Corresponding author: Smida M, Orthopedics Kassab Institute, 2010 Ksar Said La Manouba, Tunis Faculty of Medicine, Manar University, Tunisia, Tel: 0021623317136; E-mail: mahmoud.smida@yahoo.fr

Received date: May 28, 2018; Accepted date: June 22, 2018; Published date: June 29, 2018

Copyright: (c) 2018 Smida M. This is an open-access article distributed under the terms of the Creative Commons Attribution License, which permits unrestricted use, distribution, and reproduction in any medium, provided the original author and source are credited.

\begin{abstract}
Objective: The objectives of this study were to determine the vitamin D status in a sample of Tunisian children with Osgood-Schlatter disease (OSD).

Patients and methods: We performed a prospective study including normal and healthy children presented with OSD. An assessment of the status of vitamin D measuring serum concentrations of 25-hydroxy vitamin D, was done for all patients using the chemiluminescence immunoassays technique.

Results: During the study period, 80 children have been enrolled and the mean age was 13.6 years. All children had low-level vitamin $D$ with a mean of $19.2 \mathrm{ng} / \mathrm{mL}$ (range of $5-27 \mathrm{ng} / \mathrm{mL}$ ). All the children were then placed under a vitamin D substitution. At follow-up, majority of knees were asymptomatic and functioned unaltered with all activities. Radiologically, all patients showed healing of the tibial tuberosity secondary ossification center.
\end{abstract}

Conclusion: In our study, all children with OSD had low-level vitamin D and after supplementation of vitamin D, the symptoms were resolved. Vitamin D deficiency screening should be instituted among children at risk of OSD and early vitamin D supplementation should be started for prevention.

Keywords: Knee; Child; Osgood Schlatter disease; Pathophysiology; Vitamin D

\section{Introduction}

Osgood-Schlatter disease (OSD), the most common osteochondrosis in children, is a very common cause of knee pain in children. It is a well-described clinical entity characterized by pain and swelling at the anterior tibial tuberosity. Usually no specific trauma or event precedes the onset of these symptoms. The lesion is bilateral in many cases and affects more commonly boys than girls. It is seen particularly in active adolescents and is a typical sport-related complaint that affects performance and achievement. It may lead to a temporary cessation of sports activities and sometimes a premature and definitive stop of a career [1]. Its etiology remains controversial and various theories have been proposed to explain the pathogenesis of OSD. Avascular necrosis, inflammation, mechanical, hormonal or traumatic factors are advanced as causes of this lesion. Despite insufficient evidence, the theory of chronic trauma leading to a minimal avulsion fracture of the tibial tuberosity secondary ossification center, is currently widely accepted. Repeated tensile extension forces of a strong patellar tendon exerted on a weak apophysis cartilage could result in avulsion of some portions [2].

But why do only some children develop an OSD which is often unilateral? This is the main question that will be asked continually. In order to answer this question, we have discussed the possibility of a relationship between vitamin $\mathrm{D}$ and OSD. In fact, vitamin $\mathrm{D}$ is essential for calcium-phosphate homeostasis and, thus, plays a key role in bone health. Furthermore, vitamin D deficiency represents a current common problem among otherwise healthy adolescents and is incriminated as a risk factor for many musculoskeletal disorders in healthy children. The main purposes of this study were to determine the vitamin D status in a sample of Tunisian children with OSD and to investigate the relationship between vitamin D insufficiency and this osteochondrosis.

\section{Patients and Methods}

\section{Study design}

We performed a prospective study including children presented with OSD from January 2009 to December 2014. Patients were recruited through the outpatient orthopedic clinic of two hospitals. They all satisfied the following inclusion and exclusion criteria:

Inclusion criteria: Healthy children of Tunisian parents, born and living in Tunisia and presenting OSD with open physeal plate of proximal tibia.

Exclusion criteria: Congenital or acquired bone mineralization disorder (rickets, osteomalacia, osteogenesis imperfecta); chronic disease that may cause a disorder of vitamin D stock (renal failure or celiac disease or endocrinopathy); taking drugs that block the vitamin D metabolism (barbiturates, corticoids); any condition limiting physical activity; stay outside Tunisia exceeding 3 months; dietary 
vitamin D supplementation; OSD in the sequelae stage with close physeal plate of proximal tibia. The diagnosis of OSD was confirmed when a teenager had a prominent painful tibial tuberosity. Pain had to be related to direct pressure and to physical or sport activities. Fragmentation of the tibial tuberosity secondary ossification center on the knee X-rays lateral view was also required for diagnosis. Following clinical examination and X-ray exam making the diagnosis of OSD, an assessment of the status of vitamin $\mathrm{D}$ measuring serum concentrations of 25-hydroxy vitamin D, was done. No control group was recruited for this study.

In the absence of a local Institution Ethics Committees, the study was reviewed and approved by the staffs of the two originating departments.

\section{Clinical measures}

Rating of knee pain was obtained from all patients using 0-10 visual analog scale ruler (VAS).

\section{Biochemical measurements and data interpretation}

Measurement of serum 25-hydroxy vitamin D level was done in two laboratories using the chemiluminescence immunoassays technique. The interpretation of the values was discussed in an interdisciplinary meeting with one of the authors, an endocrinologist and we used the reference values proposed by the two reference laboratories as follows:
- $\quad$ vitamin D sufficient $\geq 30 \mathrm{ng} / \mathrm{mL}$.

- vitamin $\mathrm{D}$ insufficient $\geq 10 \mathrm{ng} / \mathrm{mL}$ and $<30 \mathrm{ng} / \mathrm{mL}$.

- vitamin D deficient $<10 \mathrm{ng} / \mathrm{mL}$.

\section{Statistical Analysis}

Data were analyzed using SPSS (IBM SPSS Statistics for Windows, Version 20.0. Armonk, NY: IBM Corp.). Independent t-test was used for group comparisons and a $\mathrm{p}$-value $\leq 0.05$ was considered statistically significant. Values are expressed as mean \pm SD (Standard Deviation).

\section{Results}

During the study period, 80 children have been enrolled. The mean age was 13.6 years (age range from 10 to 14.7 years) and the male to female ratio was $66 / 14$ ( $82.5 \%$ were boys). Of the 80 patients, 15 had bilateral involvement, totaling 95 knees. No female patient in the group wore enveloping clothing and Hijab, the Islamic veil that cover exposed skin of head, chest and limbs.

The initial mean VAS score was $5.47 \pm 1.04$ (range of 4-7). Data analyses showed that all children (100\%) had low-level vitamin D (25$\mathrm{OH}$ Vitamin $\mathrm{D}<30 \mathrm{ng} / \mathrm{mL}$ ) with a mean of $19.22 \pm 5.42 \mathrm{ng} / \mathrm{mL}$ (range of 5-27 ng/mL) (Table 1). Seventy-five children (93.8\%) were vitamin D insufficient with a mean of $20.03 \pm 4.53 \mathrm{ng} / \mathrm{mL}$ (range of 10.5-27 $\mathrm{ng} / \mathrm{mL}$ ) and five cases $(6.2 \%)$ were vitamin D deficient with a mean of $7 \pm 1.72 \mathrm{ng} / \mathrm{mL}$ (range of $5-9 \mathrm{ng} / \mathrm{mL}$ ).

\begin{tabular}{|l|l|l|l|}
\hline & All & Insufficient & Deficient \\
\hline Number patients (\%) & $80(100 \%)$ & $75(93.8 \%)$ & $5(6.2 \%)$ \\
\hline Age (Months) & $163.18 \pm 10.88$ & $164.2 \pm 10.38$ & $147.8 \pm 5.67$ \\
\hline Vitamin D (ng/mL) & $19.22 \pm 5.42$ & $20.03 \pm 4.053$ & $7 \pm 1.72$ \\
\hline VAS & $5.47 \pm 1.04$ & $5.45 \pm 1.05$ & $5.8 \pm 0.83$ \\
\hline
\end{tabular}

Table 1: Comparison of insufficient and deficient groups before vitamin D substitution.

Vitamin D deficient group was younger than vitamin D insufficient group with an average age of $147.8 \pm 5.67$ and $164.2 \pm 10.38$ respectively; the difference was statistically significant $(\mathrm{p}=0.001)$. Vitamin D insufficient group had a mean VAS score of $5.45 \pm 1.05$ (range of 4-7) and vitamin D deficient group had a mean VAS score of $5.8 \pm 0.83$ (range of 5-7); the difference was not statistically significant $(\mathrm{p}=0.475)$ (Table 1).

In view of these findings and after discussion with our endocrinologist, we began a treatment protocol of vitamin D substitution. After explanation of the results and the role of vitamin D, the parents were informed of the protocol aims and gave their oral consent. We agreed to start supplementation with Ergocalciferol (Sterogy $1^{\oplus} 6000$ IU per day) and a dosage of vitamin D had to be done at the disappearance of the knee pain or automatically at 6 and/or 12 months in cases of bad clinical response. This posology was continued until knee pain had resolved and vitamin D was corrected.

After starting treatment, patients were routinely followed for clinical evaluation at 1,3, 6 months, 1 year and two years. X-rays were taken at 3, 6 months and one year after. One month after, evolution was marked by significant improvement of pain in 74 children who returned to almost normal sport and play activities. Rating of pain by
VAS score was obtained from only 31 children; they had a mean score of 2.8 . At the end of 3 months of treatment, 80 knees were completely asymptomatic. In 15 knees, pain did not disappear completely, but children did their sport activities without being embarrassed. 
Citation: Smida M, Kandara H, Jlalia Z, Saied W (2018) Pathophysiology of Osgood-Schlatter Disease: Does Vitamin D have a Role?. Vitam Miner 7: 177.

Page 3 of 6

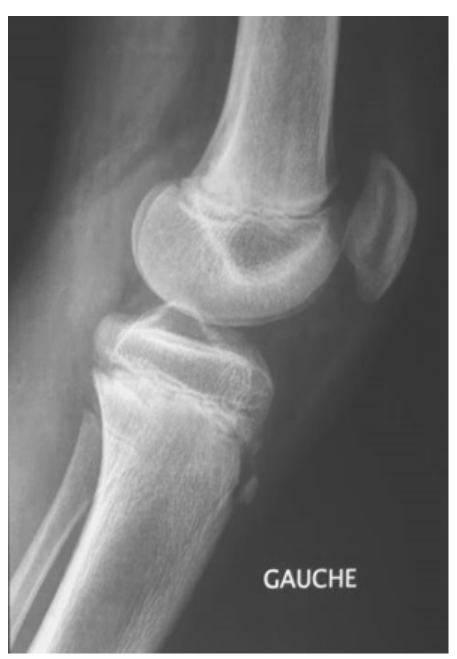

Figure 1a: Left OSD in a 12 years-old boy with tibial tuberosity fragmentation $(25[\mathrm{OH}]$ Vitamin D: $10.7 \mathrm{ng} / \mathrm{ml})$.

At 6 months and 1-year follow-up, 90 knees were completely asymptomatic. In five knees, pain occurred only after sport activities (the mean VAS score was $1.6 \pm 0.54$ ). Concerning radiological outcomes, all patients showed healing of the tibial tuberosity secondary ossification center with normal re-ossification and disappearance of fragmentation (Figures 1-3). Vitamin D substitution lasted on average 7 months and was stopped digressively when the vitamin D assay showed a normal level (the mean 25-OH Vitamin D was $38.38 \pm 5.82$ $\mathrm{ng} / \mathrm{mL}$ ). No complications of treatment were observed.

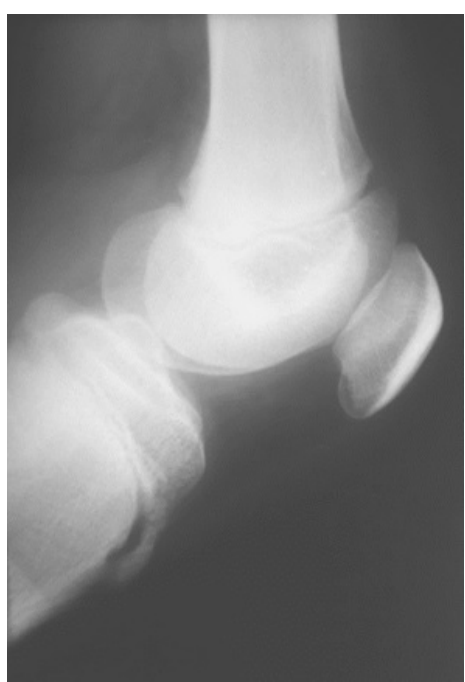

Figure 1b: The same patient: Healing of the tibial tuberosity with good mineralization after 3.5 months of vitamin $\mathrm{D}$ supplementation (25 [OH] Vitamin D: $30.21 \mathrm{ng} / \mathrm{ml})$.

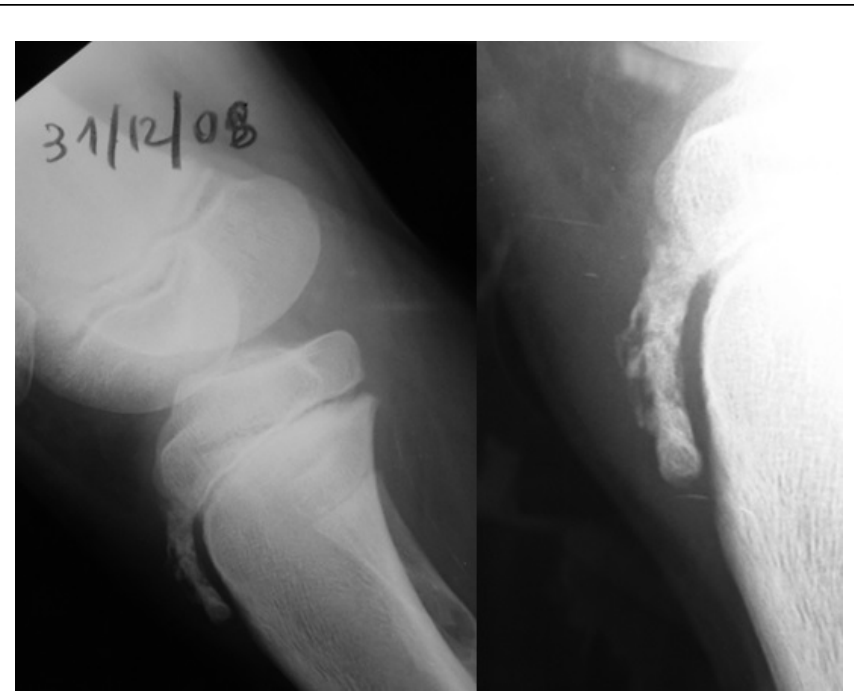

Figure 2a: Right OSD in a 13 years-old boy $(25[\mathrm{OH}]$ Vitamin D: 26 $\mathrm{ng} / \mathrm{ml})$.

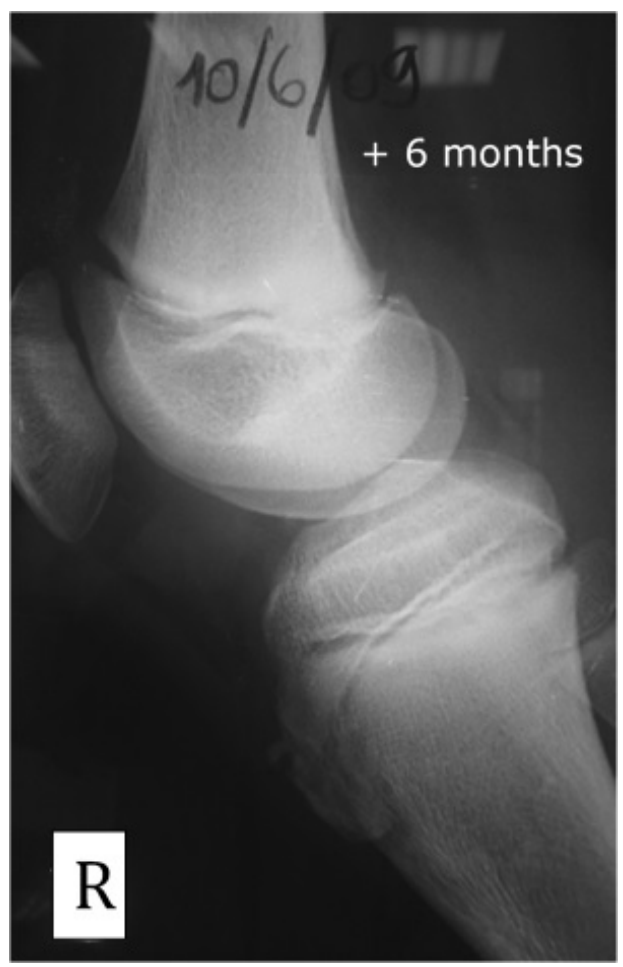

Figure 2b: The same patient 6 months after: Healing of the tibial tuberosity after vitamin D supplementation $(25[\mathrm{OH}]$ Vitamin D: $59.6 \mathrm{ng} / \mathrm{ml})$. 


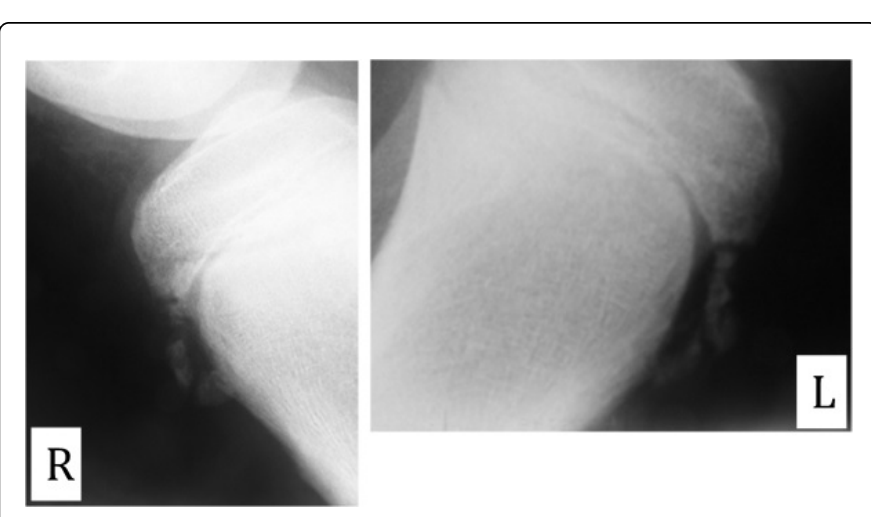

Figure 3a: Bilateral OSD in a 12 years-old boy $(25[\mathrm{OH}]$ Vitamin D: $19 \mathrm{ng} / \mathrm{ml})$.

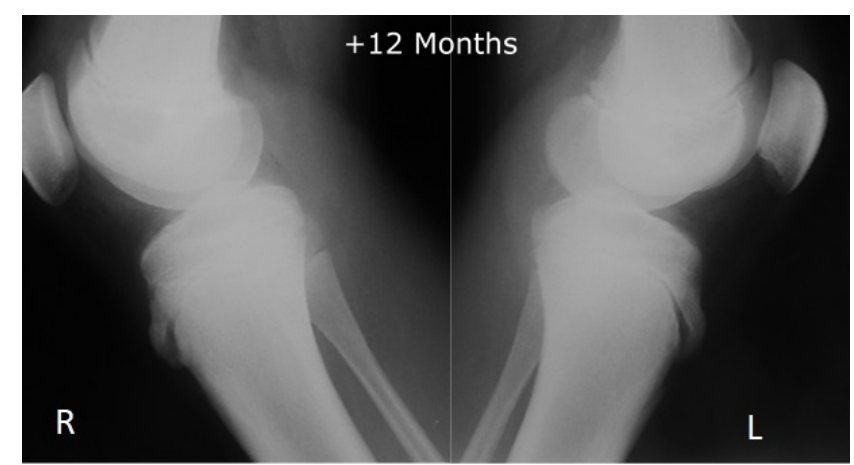

Figure 3b: The same patient 12 months after: Healing of the tibial tuberosity after vitamin D supplementation $(25[\mathrm{OH}]$ Vitamin $\mathrm{D}$ at 6 months: $36.54 \mathrm{ng} / \mathrm{ml})$.

\section{Discussion}

OSD represents a most common cause of knee pain in children in the second decade of life particularly who are practicing regular sport activities [1]. In the early stages of OSD, pain is typically exacerbated with activities and becomes permanent even without physical activities. The current standard treatment of acute and active OSD is non-operative [3]. However, in many recalcitrant cases, the conservative measures are not efficient and the impact on the daily life of the child and his parents becomes cumbersome. Moreover, 5 to $10 \%$ of patients continue to complain of local pain and restricted activity into adulthood due to an unfused ossicle, requiring then surgical treatment [4].

It is therefore very important to understand the pathophysiology of OSD and its mechanisms that lead to the disease state with its pathological lesions. Usually these mechanisms are set in motion by underlying etiological causes, which dedicate the treatment, and if controlled would allow the disease to be effectively prevented [5]. Multiple theories have been proposed and many causes have been incriminated in the genesis of OSD. But for lack of evidence, these theories were rejected except the traumatic traction theory that is currently widely accepted. The majority of authors believe now that the repetitive strain caused by the strong and violent pull of the quadriceps muscle and applied to the weak apophyseal cartilage of the tibial tuberosity, results in avulsion of segments of its anterior part [1].

Ogden and Southwick [6] suggested that OSD is secondary to the inability of the developing secondary ossification center to withstand the forces from the patellar tendon, resulting in avulsion of a portion of the developing center. Hirano et al. [1] were able to map the progress of avulsion process and they defined five stages. In the early stage, edema-like changes around the tibial tuberosity are present. Second, in the progressive stage, a partial tear in the secondary ossification center is detected and if extended, avulsion happen. In the terminal stage, the avulsed part is pulled superiorly and separated completely forming then the ossicle. The intervening region may heal subsequently by fibrocartilage that could ossify. This recovery situation indicates the healing stage.

Over time, this traumatic theory has been supported by other studies evoking mechanical causes. The length of the moment arm from the patellar tendon to the center of rotation of the knee had appeared biomechanically important [7]. The anatomical positions of the tibial tuberosity and the patella, the length of patellar tendon and its insertion are all factors that determine the moment arm. Aparicio et al. [8] and Jacob et al. [9] reported a strong association between OSD and patella alta by increasing the strain at the patellar tendon insertion. Lancourt and Cristini [10] found patella infera in a small series of adolescents with OSD before physeal closure. The low-lying patella, with shortening of the patellar tendon, may explain the increased stress on the tibial tuberosity.

The patellar tendon attachment to the tibia was also considered in biomechanical calculations. In their magnetic resonance imaging (MRI) study, Demirag et al. [11] found that the patellar tendon was attached more proximally and had broader insertion above the tibial physis in OSD. Lee et al. [12] also revealed that the patellar tendon in a group of 30 patient with OSD was attached more widely to the tibial tuberosity, resulting in a reduced free tendon portion, and more proximally to the tibial articular surface compared with a control group. In fact, more proximal attachment of the patellar tendon reduces the portion of the free tendon and so increases the effects of its forces on the tibial tuberosity by shortening of the lever arm working on the tendon and probably causes OSD [11].

Shortening of the rectus femoris was also incriminated in alteration of the biomechanical function of the knee by means of the lever arm and increased muscular strength involved in performing a knee extension [13]. Quadriceps muscle tightness and strength have been also implicated; they increase with skeletal maturation and may lead to partial avulsion of the secondary ossification center of tibial tuberosity [14]. In addition, the maturity stage of the tibial apophysosis seems to be important and most cases of OSD are seen in the period from apophyseal to epiphyseal stages $[1,3,14]$.

Knowing the important role of vitamin D in calcium homeostasis and skeletal development, we have considered a potential link between OSD and vitamin D deficiency and we started our study. Vitamin D ensures normal calcium level in the blood, which is necessary to enhance normal calcification of the growth plate and mineralization of growing bone in children [15]. For these reasons, children have higher calcium demand and vitamin $\mathrm{D}$ need than adults particularly in adolescence when growth velocity is maximal and bone mass is increased [16].

Deficiency of vitamin D leads to disruption of the organization of the growth plate [17] and impairment of mineralization in the zone of 
provisional calcification [18] and significantly causes growth retardation and development of skeletal deformities such as rickets [19]. Furthermore, vitamin D deficiency or insufficiency have been associated to many musculoskeletal disorders with a growing number of studies in recent years [20-22]. In parallel, there is now evidence indicating that vitamin $\mathrm{D}$ deficiency becomes a public health problem worldwide even in sunny countries [23,24] with an estimated prevalence of $29-100 \%$ in children and adolescents [15]. In a recent study from our country, vitamin D inadequacy was observed in $85 \%$ of 225 Tunisian children aged 7-15 years and practicing sports in a football academy [23]. However, the classification of vitamin D deficiency remains controversial and a wide variation in its definition is until now used [25]. At the 5th International Symposium on the nutritional aspects of osteoporosis in 2003, experts define the vitamin $\mathrm{D}$ deficiency by concentrations of $25 \mathrm{OH}$ vitamin $\mathrm{D}<30 \mathrm{ng} / \mathrm{ml}(75$ nmol/l) [26]. Consistently with this proposal and based on the two laboratories reference values, a level of vitamin $D$ inferior to $30 \mathrm{ng} / \mathrm{mL}$ was considered vitamin D deficiency in our study.

To the best of our knowledge, the possible association of vitamin $\mathrm{D}$ deficiency with OSD has not been considered in the past and our study is the only one and first that assessed this association. However, how we can explain this association? OSD involves the enthesis, which is a transitional tissue interface between patellar tendon and tibial apophysis. This anatomical entity is fibrocartilaginous and has many structural similarities to the growth plate with four zones and its maturation may follow a similar pattern of endochondral ossification [27]. This process of maturation leads to specific gradients in extracellular matrix structure and composition [28]. Within the transitional zone of the enthesis, an increase in mineral relative to collagen has been observed through the transition from tendon to bone [29]. These variations in structural and compositional properties result in graded mechanical behavior that contributes to an efficient transfer of muscle load from tendon to bone by limiting stress concentrations [30].

In relation with the process of mineralization, we think that vitamin $\mathrm{D}$ deficiency could lead to disturbances in mineral transition of the enthesis and mineral composition of the apophysis. Vitamin D deficiency contributes then to a decreased mechanical performance of the load-bearing interface and to a diminished resilience of the apophysis causing the lesions observed during OSD. Another main question is why OSD affect often only one apophysosis while the deficiency in vitamin $\mathrm{D}$ is general?

We believe that the cause of the OSD is multifactorial and thus suggest a multifactorial theory incriminating a general biological factor and local mechanical factors. Mechanical factors are represented by patella alta, patella infera, shortness of rectus femoris, proximal attachment and wide attachment of the patellar tendon. These predisposing local factors interact with physiological tightness of quadriceps muscle present during adolescence and increase the forces of the lever arm exerted on the tibial tuberosity. Vitamin D deficiency, the general biological factor, would aggravate the fragility of the tibial tuberosity, which is physiological at this age of transition between the apophyseal stage and the epiphyseal stage and would cause a decrease in the mechanical performance of the enthesis. Together, these mechano-physio-biological factors would lead to traumatic avulsion of the tibial tuberosity by traction of the patellar tendon and produce OSD. A single mechanical factor may be required. This mechanobiological imbalance can then solve the enigma of the occurrence of
OSD in some children and not in others, on a single knee and not both in the same patient.

In our study, all children with OSD had low-level vitamin D, and after supplementation of Vitamin D, the symptoms of OSD were resolved. These new findings have clinical and research implications. Vitamin D deficiency may play an important role in the genesis of OSD. The clinical implication is that vitamin D deficiency screening should be instituted among children at risk of OSD and early vitamin D supplementation should be started for prevention. The important research implication is that additional randomized controlled trials should be performed to confirm our results. Given the efficiency of vitamin $\mathrm{D}$ supplementation demonstrated in our OSD group, and because of its easy use, good tolerance and safety, it should be used in other apophysosis, such as Sever disease. Further future research studies are needed.

Our study has many limitations and the absence of a control group is the significant limitation. In addition, physiological seasonal variations in vitamin $\mathrm{D}$ values were not considered. Moreover, we do not investigate anthropometry and other predisposing factors of OSD such as tightness of the quadriceps femoris and length of patellar tendon.

\section{Funding}

None.

\section{Conflict interest}

The authors declare that they have no conflict of interest.

\section{Acknowledgments}

We thank Professor Mohamed Ridha Cherif for allowing us to do a part of this study in the Orthopedic Department of Bizerte. We thank also Professor Nissaf Ben Alaya for statistical analysis.

\section{References}

1. Hirano A, Fukubayashi T, Ishii T, Ochiai N (2002) Magnetic resonance imaging of Osgood-Schlatter disease: the course of the disease. Skeletal Radiol 31: 334-342.

2. Rosenberg ZS, Kawelblum M, Cheung YY, Beltran J, Lehman WB, et al. (1992) Osgood-Schlatter lesion: Fracture or tendonitis? Scintigraphic, CT, and MR Imaging features. Radiology 185: 853-858.

3. Yanagisawa S, Osawa T, Saito K, Kobayashi T, Tajika T, et al. (2014) Assessment of Osgood-Schlatter disease and the skeletal maturation of the distal attachment of the patellar tendon in preadolescent males. Orthop J Sports Med 2: 2325967114542084.

4. Weiss JM, Jordan SS, Andersen JS, Lee BM, Kocher M (2007) Surgical treatment of unresolved Osgood-Schlatter disease: ossicle resection with tibial tubercleplasty. J Pediatr Orthop 27: 844-847.

5. Bahr R, Krosshaug $\mathrm{T}$ (2005) Understanding injury mechanisms: a key component of preventing injuries in sport. Br J Sports Med 39: 324-329.

6. Ogden JA, Southwick WO (1976) Osgood-Schlatter's disease and tibial tuberosity development. Clin Orthop Relat Res 116: 180-189.

7. Kaufer H (1971) Mechanical function of the patella. J Bone Joint Surg Am 53: 1551-1560.

8. Aparicio G, Abril JC, Calvo E, Alvarez L (1997) Radiologic study of patellar height in Osgood-Schlatter disease. J Pediatr Orthop 17: 63-66.

9. Jacob RP, Von Gumppenberg S, Engelhard P (1981) Does OsgoodSchlatter disease influence the position of the patella? J Bone Joint Surg B 63: $579-582$. 
10. Lancourt JE, Cristini JA (1975) Patella alta and Patella infera. J Bone Joint Surg Am 57: 1112-1115.

11. Demirag B, Ozturk C, Yazici Z, Sarisozen B (2004) The pathophysiology of Osgood-Schlatter disease: a magnetic resonance investigation. J Pediatr Orthop B 13: 379-382.

12. Lee DW, Kim MJ, Kim WJ, Ha JK, Kim JG (2016) Correlation between magnetic resonance imaging characteristics of the patellar tendon and clinical scores in Osgood-Schlatter disease. Knee Surg Relat Res 28: 62-67.

13. de Lucena GL, dos Santos Gomes C, Guerra RO (2011) Prevalence and associated factors of Osgood-Schlatter syndrome in a population-based sample of Brazilian adolescents. Am J Sports Med 39: 415-420.

14. Nakase J, Aiba T, Goshima K, Takahashi R, Toratani T, et al. (2014) Relationship between the skeletal maturation of the distal attachment of the patellar tendon and physical features in preadolescent male football players. Knee Surg Sports Traumatol Arthrosc 22: 195-199.

15. Shin YH, Shin HJ, Lee YJ (2013) Vitamin D status and childhood health. Korean J Pediatr 56: 417-423.

16. Kumar J, Muntner P, Kaskel FJ, Hailpern SM, Melamed ML (2009) Prevalence and associations of 25-hydroxyvitamin D deficiency in US children: NHANES 2001-2004. Pediatrics 124: e362-370.

17. Sevenler D, Buckley MR, Kim G, van der Meulen MC, Cohen I, et al. (2013) Spatial periodicity in growth plate shear mechanical properties is disrupted by vitamin D deficiency. Biomech 46: 1597-1603.

18. Judd J, Welch R, Clarke A, Reading IC, Clarke NM (2016) Vitamin D deficiency in slipped upper femoral epiphysis: time to physeal fusion. J Pediatr Orthop 36: 247-252.

19. Boot AM, Krenning EP, de Muinck Keizer-Schrama SM (2011) The relation between 25 -hydroxyvitamin $\mathrm{D}$ with peak bone mineral density and body composition in healthy young adults. J Pediatr Endocrinol Metab 24: 355-360.

20. James JR, Massey PA, Hollister AM, Greber EM (2013) Prevalence of hypovitaminosis D among children with upper extremity fractures. J Pediatr Orthop 33: 159-162
21. Madhuri V, Arora SK, Dutt V (2013) Slipped capital femoral epiphysis associated with vitamin D deficiency. Bone Joint J 95: 851-854.

22. Ryan LM, Brandoli C, Freishtat RJ, Wright JL, Tosi L, et al. (2010) Prevalence of vitamin D insufficiency in African American children with forearm fractures: a preliminary study. J Pediatr Orthop 30: 106-109.

23. Bezrati I, Ben Fradj MK, Ouerghi N, Feki M, Chaouachi A, et al. (2016) Vitamin D inadequacy is widespread in Tunisian active boys and is related to diet but not to adiposity or insulin resistance. Libyan J Med 11: 31258.

24. Wakayo T, Belachew T, Vatanparast H, Whiting SJ (2015) Vitamin D deficiency and its predictors in a country with thirteen months of sunshine: the case of school children in central Ethiopia. PLoS One 10: e0120963.

25. Bischoff-Ferrari HA, Giovannucci E, Willett WC, Dietrich T, DawsonHughes B (2006) Estimation of optimal serum concentrations of 25hydroxyvitamin D for multiple health outcomes. Am J Clin Nutr 84: 18-28.

26. Dawson-Hughes B, Heaney RP, Holick MF, Lips P, Meunier PJ, et al. (2005) Estimates of optimal vitamin D status. Osteoporos Int 16: 713-716.

27. Zelzer E, Blitz E, Killian ML, Thomopoulos S (2014) Tendon-to-bone attachment: from development to maturity. Birth Defects Res C Embryo Today 102: 101-112.

28. Blitz E, Viukov S, Sharir A, Shwartz Y, Galloway JL, et al. (2009) Bone ridge patterning during musculoskeletal assembly is mediated through SCX regulation of Bmp4 at the tendon-skeleton junction. Dev Cell 17: 861-873.

29. Wopenka B, Kent A, Pasteris JD, Yoon Y, Thomopoulos S (2008) The tendon to bone transition of the rotator cuff: a preliminary Raman spectroscopic study documenting the gradual mineralization across the insertion in rat tissue samples. Appl Spectrosc 62: 1285-1294.

30. Cooper RR, Misol S (1970) Tendon and ligament insertion. A light and electron microscopic study. J Bone Joint Surg Am 52: 1-20. 